\title{
X-ray Variability and the Secondary Star
}

\author{
M. F. Corcoran and K. Ishibashi
}

\begin{abstract}
We discuss the history of X-ray observations of the $\eta$ Car system, concentrating on the periodic variability discovered in the 1990s. We discuss the interpretation of these variations, concentrating on a model of the system as a "collidingwind" binary. This interpretation allows the physical and orbital parameters of $\eta$ Car and its companion star to be constrained.
\end{abstract}

\section{The 5.5 Year Period}

Periodic variability is a key diagnostic in astrophysics. Astronomers long sought periodicities in $\eta$ Car's behavior ever since its giant eruption. Before 1870 Rudolf Wolf and Elias Loomis suggested photometric cycles of 46 and 67 years, respectively $[99,60]$; a century later Payne-Gaposchkin proposed a cycle of 15-16 years [73], and Feinstein \& Marraco suspected a 3-year periodicity [35]. The complex behavior of the observed variations from $\eta$ Car in broad-band photometry and in spectrographic observations made determination of the true period difficult.

The discovery of $\eta$ Car's first confirmed period depended on observations spanning twenty years or more. This began with the recognition of a so-called "spectroscopic event" by Zanella, Wolf and Stahl in 1981[100, 95]. These "events" represent a dramatic and complex change in $\eta$ Car's visible-band spectrum in which high-excitation He I and [Ne III] emission lines abruptly fade, then recover. Similar changes happened in 1948 and 1965, which suggested to them a recurrence

M. F. Corcoran

Center for Research and Exploration in Space Science \& Technology, Code 662, Goddard Space Flight Center, Greenbelt, MD, 20771 USA and Universities Space Research Association, 10211 Wincopin Circle, Suite 500, Columbia, MD, 21044 USA. e-mail: Michael.F.Corcoran@ nasa.gov

Kazunori Ishibashi

Nagoya University, Department of Physics, 1 Furo-cho Chikusa-ku, Nagoya, Aichi, Japan 4648602. e-mail: bish@u.phys.nagoya-u.ac.jp 
timescale of 17 years [100]. They were on the right track, but did not know that other such events had occurred (as we deduce today) in 1953,1959, 1970, and 1975. Near-infrared photometry from South Africa obtained by Patricia Whitelock and her collaborators[96] over 156 nights in 1975-1994 showed a significant 5-6 year cycle, but this cycle was confused with other near-infrared variations on other timescales. Meanwhile new spectroscopic events occurred in 1986 and in 1992, though the former was not widely reported.

The breakthrough was provided by a set of 27 measurements of the He I $10830 \AA$ emission line obtained by Augusto Damineli at Brazil's National Astrophysical Laboratory. These observations, augmented by 4 additional measures from other observers from 1981-1995, showed a clear, rapid weakening of this He I line every 5.5 years, lasting only for a few weeks (a duty cycle of $<5 \%$ ). Damineli showed[16] the fading of the He I 10830 line occurred at nearly the same time as brightening of the system in the near IR, and that the 5.5 year period recovered not only the "spectroscopic event" seen by Zanella, Wolf and Stahl in 1981, but other "spectroscopic events" observed in the previous 50 years. Damineli successfully predicted the occurrence of the next minimum at the end of 1997.

Thanks to multi-wavelength observations of $\eta$ Car during the subsequent minima in December 1997 and June 2003, we know that the fading occurs throughout nearly the entire electromagnetic spectrum from the radio through the IR, optical, UV and $\mathrm{X}$-ray region (the situation in the (amma-ray band is less clear). The period is almost exactly the same in every wavelength region[21]. Detailed monitoring observations since 1997 have refined the period to 2024 days $(5.54 \mathrm{yr}$ ) with an uncertainty that appears to be no more than a week and may be as small as \pm 2 days[20].

What regulates the cycle? Following Zanella et al., Damineli initially supposed that each spectroscopic event is a single-star "shell ejection", a temporary large increase in the stellar mass-loss rate, reminiscent of phenomena seen in S Dor variables and Luminous Blue Variable stars (see, e.g., [46]). But $\eta$ Car's variability cycle is too long to represent a pulsation or rotation period, since those periods should be only days or weeks. A thermal/rotational recovery cycle could have a timescale of several years [31], but this seems unlikely to produce so regular a period. The He I 10830 variation seems strictly periodic and strongly suggests a simple clocklike explanation: a companion star in orbit around $\eta$ Car. If the 5.54 year period is assumed to be the orbital period of the system, the high total mass (probably 150 to $200 M_{\odot}$ ) implies a semi-major axis close to $17 \mathrm{AU}$. The short duty cycle implies that the phenomenon occurs near periastron passage in a highly eccentric orbit. Proposed orbits $[17,18,23,24,25,26,53]$ are mostly comparable in size and shape to the orbit of Halley's Comet.

But the UV-to-IR emission regions include both the wind of $\eta$ Car and the detritus of the eruptions from the $19^{\text {th }}$ century and are spatially complex. Emission from one region may be absorbed by cold gas in another. Dense clouds may shadow one parcel of gas from the photospheric radiation but not its neighbor. More distant gas reacts more slowly to changes in the inner system because of light-travel time, which may be days or even weeks. These complications make interpreting the spectrum at these wavelengths difficult. 
A binary system composed of luminous stars must produce significant amounts of X-ray emission resulting from the high speed collision of the massive stellar winds $[5,76]$ which constantly flow off the stellar surfaces. This wind-wind collision will produce a "bow shock" where the wind kinetic energy is converted to thermal energy which radiates mostly in the X-ray band (as discussed below). Such emission is an important probe of massive binaries since it originates in a localized region (defined by system parameters like the separation between the two stars and the relative mass loss rates and wind speeds), and it penetrates through large columns of intervening cold material. What do X-ray observations tell us about $\eta \mathrm{Car}$ ?

\section{X-ray Emission and Variability}

Studies of X-ray emission from $\eta$ Car go back 30 years or more, nearly to the beginning of $X$-ray astronomy. The earliest data were obtained by non-imaging detectors with fields of view defined by collimators, flown on rockets or orbiting platforms $[42,39,36,77,69]$. They established $\eta$ Car as a source of X-rays at energies $E>5$ $\mathrm{keV}$, and detected iron $\mathrm{K}$-line emission at $6.7 \mathrm{keV}$. They also provided hints of variability, though direct comparisons of X-ray flux were hampered by differences in instrumentation (fields of view, sensitivities, etc).

Today we know that many $X$-ray sources, both discrete and diffuse, exist near $\eta$ Car, so it was not until the advent of imaging $X$-ray telescopes that $X$-ray emission from $\eta$ Car could be cleanly disentangled from this contamination. The first X-ray images of $\eta$ Car and the Carina Nebula were obtained in the early 1980s with the HEAO-2/Einstein satellite observatory $[78,79,6]$. Those observations showed that at energies above $3 \mathrm{keV}, \eta$ Car was the only source visible in the field. They showed that $X$-ray emission from $\eta$ Car consists of at least two components: an extended $\left(\sim 20^{\prime \prime}\right)$ "soft" $(E<1 \mathrm{keV})$ component (likely produced by the collision of material from the 1840 's eruption with interstellar material) surrounding a point-like, "hard" $(E>3 \mathrm{keV}$ ) heavily absorbed component centered on the optical position of $\eta$ Car. This "point-like" component has a temperature of $4-8 \times 10^{7} \mathrm{~K}$ and its X-ray emission is absorbed by intervening material having a column density of $5-50 \times 10^{22}$ atoms $\mathrm{cm}^{-2}[6,12,40]$. The observed luminosity of the hard, point-like source measured by Einstein (adjusted to the current best estimated distance of $2300 \mathrm{pc}$ ) was $\approx 4 \times 10^{33} \mathrm{ergs} \mathrm{s}^{-1}$ in the $0.2-4 \mathrm{keV}$ band; correcting for absorption, the luminosity of the source is about $2 \times 10^{34} \mathrm{erg} \mathrm{s}^{-1}$. While these observations were key to establishing the overall character of the high-energy $\mathrm{X}$-ray emission from $\eta$ Car, these relatively short "snapshots" could not really address issues of temporal variability.

Figure 1 shows a Hubble Space Telescope image of $\eta$ Car and the Homunculus nebula superimposed on a more recent $X$-ray image from the Chandra $X$-ray Observatory. This image clearly shows the extended "shell" of X-ray emission first seen by Einstein surrounding the Homunculus along with the point-like $\mathrm{X}$-ray emission coincident with the optical position of the star. The point-like, hard emission was initially interpreted $[79,6]$ as the collision of a fast wind from $\eta$ Car with a circum- 
stellar "shell" about 1000 AU from the star. As we shall see, the temperature of the emission requires a wind velocity of $2000-3000 \mathrm{~km} \mathrm{~s}^{-1}$ or more if it's solely produced by conversion of the wind kinetic energy to heat. This is about 4 times faster than $\eta$ Car's bulk wind seen in visual-wavelength spectra - a highly signficant point in retrospect, though it was not emphasized at the time of the Einstein observations. The X-ray absorbing column depth was thought to be consistent with absorption in a 3 solar mass cold shell, roughly agreeing with what was known of the mass of the Homunculus at that time.

\subsection{Discovery of $X$-ray Variability}

$X$-ray variability of $\eta$ Car was first detected by the ROSAT X-ray observatory in short observations obtained in 1992-1993 analyzed by G. L. Rawley. She showed that the central source was not visible in June and August of 1992 at energies above $2 \mathrm{keV}$, but was clearly detected in January 1993|7|. The flux above $2 \mathrm{keV}$ varied by a factor of two in a 4-month interval, requiring a tripling of the mass-loss rate if the $\mathrm{X}$-rays were produced by collision of the wind with a circumstellar shell. However, variations in the temperature of the emission or changes in the intervening absorbing medium could not be ruled out.

Luck often plays a role in astronomical discovery; the mid-1992 ROSAT observations just happened to coincide with one of the spectroscopic events, and to coincide with the first observations of the star[33] by the radio telescopes of the Australia Telescope National Facility. At the same time $\eta$ Car faded in the hard X-ray band, it also faded in the radio [33], but brightened in the near-IR [96, 16]. Thus, when the 5.5-year period was identified by Damineli shortly thereafter, an obvious question was whether X-ray variability was another aspect of the same cycle.

\subsection{The X-ray Variability Cycle}

Monitoring observations with the Proportional Counter Array (PCA) on the Rossi $X$ ray Timing Explorer (RXTE) [4] provided the next X-ray breakthrough. Unlike other $X$-ray observatories, RXTE can view $\eta$ Car at any time of the year making it a nearideal instrument to study the variability of the $2-10 \mathrm{keV}$ flux. RXTE observations of $\eta$ Car began in February 1996 and have continued through 2009[52, 14]. Ty pically RXTE obtained 2-4 observations of $\eta$ Car per month, with daily observations during important intervals. The RXTE X-ray count rates vs. time are shown in figure 2.

The RXTE observations show the important temporal signatures of the 2024day X-ray variability cycle. For about four years out of the 5.5 year cycle, the 2$10 \mathrm{keV} \mathrm{X}$-ray luminosity is in a "quiescent" state, relatively constant at a level of $\sim 4 \times 10^{34} \mathrm{ergs} \mathrm{s}^{-1}$, but with erratic small-amplitude variations. About a year before the fading of the He I 10830 line, both the intensity and the irregular variability 

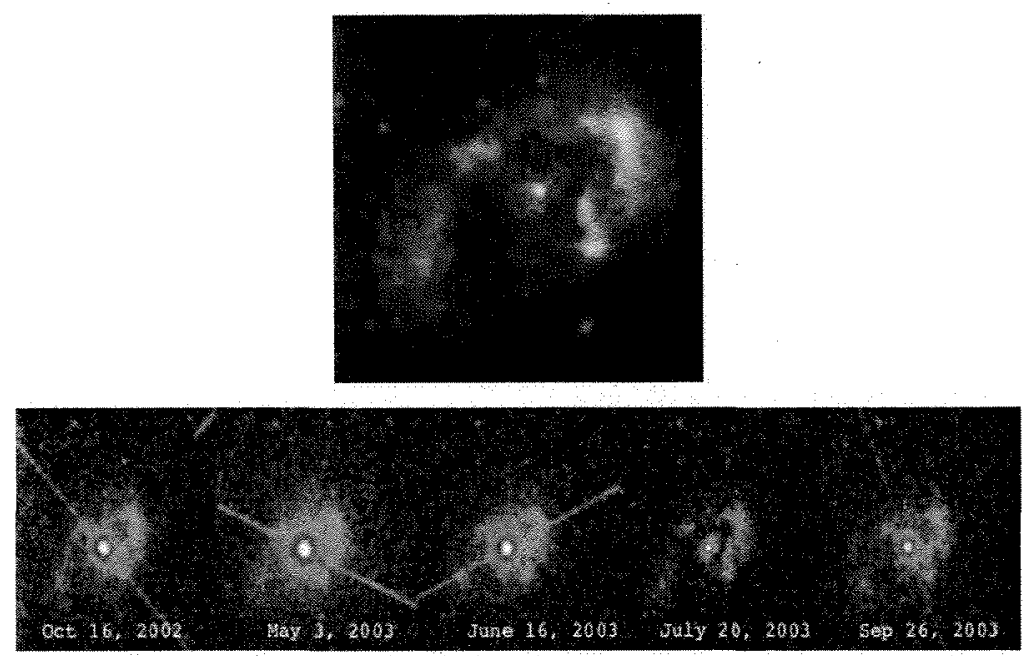

Fig. $1 \eta$ Car in X-rays. The upper figure shows an X-ray image from the Chandra X-ray observatory (in green) combined with an optical image from the Hubble Space Telescope (in red). The unresolved white point source at the center of the image, at the position of the optical star, is the source of hard X-ray emissions. The lower figure shows five Chandra ACIS-I images of $\eta$ Car, highlighting the variation of the central pointlike source. The streaks at various angles to the central source are detector artifacts.

progressively grow, and we see conspicuous X-ray "flares" with durations of weeks or days, typically two or three months apart. At the end of this phase, the average $\mathrm{X}$-ray luminosity in the $2-10 \mathrm{keV}$ band has roughly doubled to reach a maximum luminosity of $\approx 10^{35} \mathrm{ergs}^{-1}$, while the interval between flare peaks drops to less than 1 month. About 40 days after reaching its maximum, the emission swiftly (with a few weeks) falls to a level below RXTE"s detectability. This rapid decline occurs along with similarly rapid fadings of the He I 10830 line strength[21] and the IR brightness [97]. The X-ray emission usually remains in this "low state" for about 3 months. At this time the $\mathrm{X}$-ray intensity is lower than or near the cosmic $\mathrm{X}$-ray background in the $\sim 1^{\circ}$ PCA field of view, though $\eta$ Car is still detectable throughout the minimum by other X-ray observatories with sufficient spatial sensitivity [13,40] at luminosities ranging from $\sim 6 \times 10^{32} \mathrm{ergs} \mathrm{s}^{-1}$ to $5 \times 10^{33} \mathrm{ergs} \mathrm{s}^{-1}$ near the end of the minimum[40]. At the end of the low state the X-ray intensity rises rapidly to the "quiescent" level.

$\mathrm{X}$-ray spectra of $\eta$ Car show that the observable emission associated with the star is mostly in the $2-10 \mathrm{keV}$ band, corresponding to a temperature range $T=E / k$ of roughly $20-110$ million $\mathrm{K}$. The PCA spectra show little flux above $10 \mathrm{keV}$ at any phase. Near X-ray minimum, the observed emission declines more at lower energies 


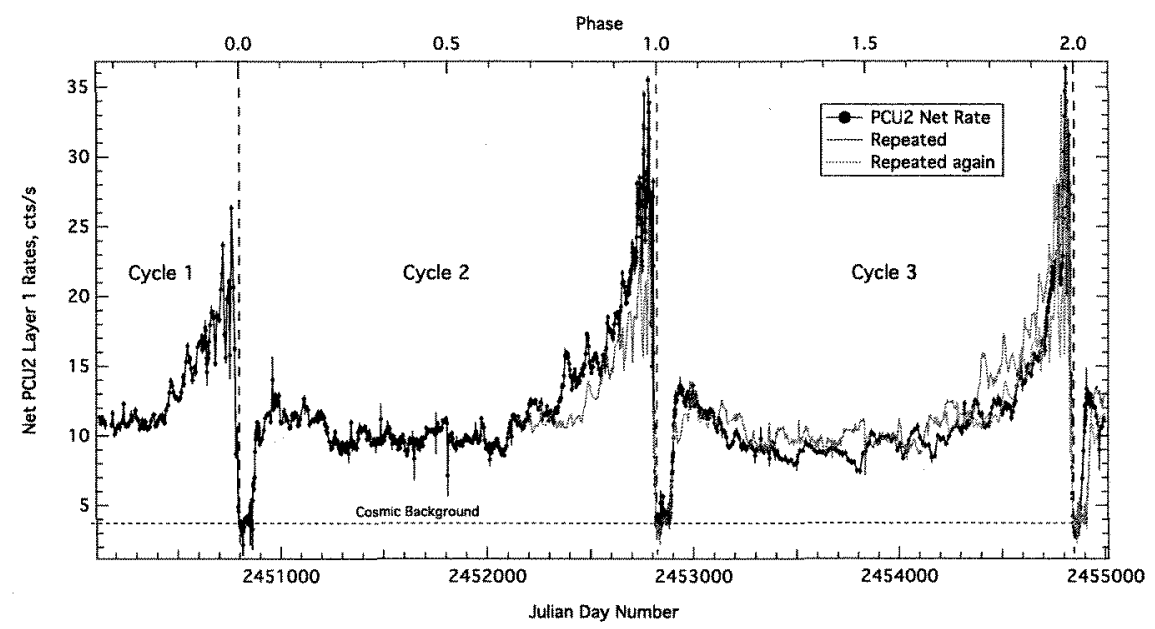

Fig. 2 The RXTE X-ray "lightcurve" of $\eta$ Car at energies above $2 \mathrm{keV}$ and below $10 \mathrm{keV}$, as measured by the PCA on RXTE. Three X-ray minima can be clearly seen near phases $0.0,1.0$ and 2.0. The "flat" bottom of the X-ray minimum seen by $R X T E$ is produced when the $X$-ray flux from $\eta$ Car falls below the cosmic background level (shown by the horizontal dashed line). The blue and red lines show the RXTE measures offset by 2024 and 4048 days, respectively.Adapted from Corcoran et al. $\lfloor 15]$.

showing the build up of circumstellar absorption, and the spectrum remains quite hard for an extended period even after the minimum ends.

\section{Colliding Wind Emission as a Diagnostic of Massive Binary Systems}

If the pan-chromatic periodicity is produced by the orbit of a companion star around $\eta$ Car (as seems likely) then this offers the unique opportunity to dynamically measure the mass of a star near the Eddington Limit using conventional binary-star radial velocity analysis. Unfortunately attempts to solve the radial velocity curves of observable emission lines in the UV-to-IR $[17,23,19,27,68]$ have not given trustworthy orbital elements, mostly because these lines are formed in a spatially complex mass flow and consequently are broad, asymmetric, variable, and highly model-dependent. For this reason, the $\mathrm{X}$-ray variability, produced by the orbit-driven variation in the wind-wind collision zone, provides a very useful (though indirect) diagnostic of the orbit. We briefly review the physics of X-ray generation in colliding wind binaries to see why this is so.

Colliding wind binaries (CWBs) produce thermal $\mathrm{X}$-rays in the strong shock where the stellar winds collide. The relevant geometry is crudely sketched in Figure 3. The shock is concave toward the star with the "weaker" wind, i.e. the star with 
the lower wind momentum flux. The vertex of this shock, where the winds collide head-on close to the line of centers, is the "stagnation point" where the winds are fully decelerated. In the idealized case a contact discontinuity separates the shocked stellar winds from the primary star on one side and secondary star on the other. The temperature of the shocked gas on each side of the contact discontinuity is to first order determined by the pre-shock velocity of the stellar wind from the star on that side. This is important for $\eta$ Car since the wind speed of $\eta$ Car itself is only $\sim 500 \mathrm{~km} \mathrm{~s}^{-1}$, which would produce only soft X-rays which would be unobservable due to heavy absorption by the cold gas in the Homunculus. The fact that hard Xrays are observed from $\eta$ Car indicates the presence of a fast flow in the system. The main X-ray emission processes are thermal Bremsstrahlung from hot electrons, and inner-shell $(\mathrm{K}, \mathrm{L}, \mathrm{M})$ transitions in nearly stripped ions. Standard examples of CWBs are HD 193793/WR 140, $\gamma^{2}$ Velorum, and HD 5980 in the SMC. These massive binaries have observed $X$-ray luminosities in the range $10^{32}-10^{36} \mathrm{ergs} \mathrm{s}^{-1}$ $\approx 0.02-300 L_{\odot}$, varying on orbital timescales. Though large, these luminosities are of course only a millionth or so of the immense stellar bolometric luminosity.

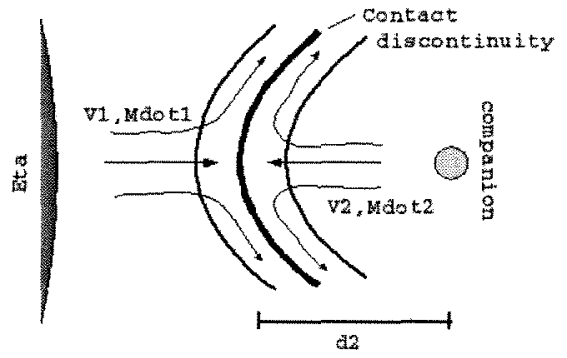

Fig. 3 A sketch of the $\eta$ Car colliding wind binary system. Winds from two stars flowing radially from the stellar surface collide and form shocks separated by a contact discontinuity. In this simple model, the shocked gas forms two curved shock fronts between which the heated plasma flows out along the contact discontinuity. Temperatures on either side of the contact discontinuity are determined by the velocities of the stellar winds just before the collision.

Theoretical descriptions of X-ray emission from wind-wind collisions in massive binaries go back to the 1970's, and the details can be quite complex [5, 76]. The essential behavior, however, can be roughly described by the following orderof-magnitude analysis by K. Davidson (private communication). Shocked gas cools by expansion and by emission of radiation as it flows outward away from the stagnation point. Characteristic flow speeds in that region should be comparable to the isothermal sound speed $w=(P / \rho)^{1 / 2} \propto T^{1 / 2}$. Based on Fig. 3, we also expect the lateral radius of the hottest region to be similar to $d_{2}$, the distance between the secondary star and the stagnation point. Therefore the expansion cooling time is

$$
t_{\mathrm{exp}} \approx \frac{d_{2}}{w} \approx \frac{d_{2}}{\left(10^{4} \mathrm{~cm} \mathrm{~s}^{-1} \mathrm{~K}^{-1 / 2}\right) T^{1 / 2}} .
$$


Meanwhile the radiative cooling time is approximately the thermal energy per unit volume divided by the Bremsstrahlung emission rate per unit volume:

$$
t_{\mathrm{rad}} \approx \frac{3 n_{e} k T}{A T^{1 / 2} n_{e}^{2}} \approx \frac{C T^{1 / 2}}{n_{e}}
$$

where $n_{e}$ is electron density, $A \approx 10^{-27} \mathrm{erg} \mathrm{cm}^{3} \mathrm{~s}^{-1} \mathrm{~K}^{-1 / 2}$, and $C \approx 10^{11.5} \mathrm{~cm}^{-3} \mathrm{~s} \mathrm{~K}^{-1 / 2}$. Thus

$$
\frac{t_{\mathrm{exp}}}{t_{\text {rad }}} \approx\left(10^{-15.5} \mathrm{~cm}^{2} \mathrm{~K}\right) \frac{n_{e} d_{2}}{T} \text {. }
$$

We can relate $T$ and $n_{e}$ in the hot shocked gas to the speed and mass loss rate in the secondary wind. According to the Rankine-Hugoniot adiabatic jump conditions across a shock $[58,80]$, the maximum speed of sound and maximum temperature at the shock front are

$$
w_{\max }=(3 / 16)^{1 / 2} v \quad \text { and } \quad T_{\max }=\frac{\mu m_{H}}{k} w_{\max }^{2},
$$

where $v$ is the pre-shock wind velocity, $m_{H}$ is the proton mass and $\mu$ is between 0.6 and 1.0 for the expected range of chemical composition. If the relevant average $T$ in the above equations is half the maximum temperature, we find

$$
T \approx\left(10^{7} \mathrm{~K}\right)\left(\frac{v}{1000 \mathrm{~km} \mathrm{~s}^{-1}}\right)^{2}
$$

As noted above, this is the reason why $\eta$ Car's primary wind does not produce observable X-rays: its typical speeds (which, it turns out, are latitudinally dependent[81]) of $200-1000 \mathrm{~km} \mathrm{~s}^{-1}$ lead to average temperatures of only a few million $\mathrm{K}$, and the resulting soft $\mathrm{X}$-rays $(E<0.7 \mathrm{keV})$ are absorbed by the large column of gas $\left(N_{H} \sim 5 \times 10^{22} \mathrm{~cm}^{-2}\right)$ in the Homunculus nebula. The observed 2$10 \mathrm{keV} \mathrm{X}$-ray spectrum indicates $T>4 \times 10^{7} \mathrm{~K}$, which requires $v>2000 \mathrm{~km} \mathrm{~s}^{-1}$. So far as observable $\mathrm{X}$-rays are concerned, $\eta$ Car's dense primary wind merely provides a wall for the secondary wind to collide into.

The jump conditions across the shock require that $n_{e}$ in the shocked gas is about 4 times as large as the electron density of the pre-shock wind. The pre-shock density is related to the secondary star's mass-loss rate by mass conservation: $\dot{M}=4 \pi r^{2} \rho \nu$. If the distance $r$ from the secondary star to the shock is roughly equal to $d_{2}$, and the pre-shock velocity of the secondary's wind is $v_{2}$, then the density in the heated gas is

$$
n_{e} \approx \frac{\left(10^{23.2} \mathrm{~g}^{-1}\right) \dot{M}_{2}}{d_{2}^{2} v_{2}}
$$

Substituting for $n_{e}$ and $v_{2}$ in the earlier expression for $t_{\exp } / t_{\mathrm{rad}}$, we find

$$
\frac{t_{\mathrm{exp}}}{t_{\mathrm{rad}}} \sim 10^{7}\left(\frac{\dot{M}_{2}}{M_{\odot} \mathrm{yr}^{-1}}\right)\left(\frac{1 \mathrm{AU}}{d_{2}}\right)\left(\frac{10^{6} \mathrm{~K}}{T}\right)^{3 / 2} .
$$


Using $\dot{M}_{2} \sim 10^{-5.5} M_{\odot} \mathrm{yr}^{-1}[74], d_{2} \sim 1$ AU near periastron, and $T \sim 5 \times 10^{7} \mathrm{~K}$ (consistent with the observed $2-10 \mathrm{keV}$ continuum), yields $t_{\exp } / t_{\text {rad }} \sim 0.1$. This means that expansion dominates the total cooling rate but not by an enormous factor.

More formal and detailed analyses of colliding winds have been calculated $[86$, $92,74]$, but the essential physics is captured by the simple analysis above. Numerical gas-dynamic simulations confirm our simplified reasoning in an order-of-magnitude sense, though incipient instabilities cause the shocked region to become spatially complex [74]. These detailed analyses suggest that the observed X-ray spectrum of $\eta$ Car requires $v_{2} \approx 3000 \mathrm{~km} \mathrm{~s}^{-1}$ because of rapid cooling of the hot gas [74].

If $t_{\mathrm{exp}} / t_{\mathrm{rad}} \gtrsim 1$ radiative cooling dominates, and the shock structure becomes unstable [86]. For $\eta$ Car this is expected to occur near periastron in the shocked primary-wind [74] on the left side of Fig. 3. In some proposed scenarios it occurs on both sides of the contact discontinuity, so that both shocks collapse near periastron $[30,61]$. In this connection it is worth noting that here we have ignored likely inhomogeneities, which may exist in the wind or may be caused by shock instabilities. If the density is inhomogeneous, then the meaning of $n_{e}$ in the expression for $t_{\exp } / t_{\text {rad }}$ becomes less obvious. On average, inhomogeneities tend to increase the fraction of input energy that is radiated.

If the ratio $\zeta=t_{\exp } / t_{\text {rad }}$ is small, then it approximately represents the efficiency for conversion of the secondary wind's kinetic energy to radiation. We noted above that the observable X-rays $(E>1 \mathrm{keV})$ in a CWB model for $\eta$ Car are powered by the companion star's fast wind, whose energy outflow rate is $\dot{M}_{2} v_{2}^{2} / 2$. Consequently the observed $\mathrm{X}$-ray luminosity should obey these approximate proportionalities:

$$
L_{x} \propto \zeta \times M_{2} v_{2}^{2} \propto \frac{\dot{M}_{2}}{d_{2} T^{3 / 2}} \times \dot{M}_{2} v_{2}^{2} \propto \frac{\dot{M}_{2}^{2}}{d_{2} v_{2}} .
$$

assuming that a constant fraction of the wind encounters the shock.

A key point is the inverse dependence of $L_{x}$ on $d_{2}$; if the companion star follows an eccentric orbit, then the varying distance modulates the X-ray luminosity such that the intrinsic $X$-ray luminosity emitted by the shock is highest near periastron and lowest at apastron. Therefore the modulated intrinsic $X$-ray luminosity can in principle be used reconstruct the orbit by determining the stellar separation as a function of time. However it is often non-trivial to derive the intrinsic X-ray luminosity from the observed luminosity, which includes the effects of variable absorption from one (or both) of the highly structured stellar winds. If we exclude the complicated interval near periastron, the apparent flux variation shown in Fig. 2 is dominated by the combination of the $1 / d_{2}$ variation of the intrinsic flux and the phase-dependent absorption along our line of sight. These two effects depend, in turn, on three classical binary-star parameters: (1) the inclination $i$ of the orbit plane, usually assumed close to $45^{\circ}$, so that the orbital angular momentum is co-linear with the bipolar axis of symmetry of the Homunculus nebula [29]; (2) the orbit eccentricity $e$, which determines $r(t)$; (3) the azimuthal orientation of the orbit, specified by the longitude of periastron $\omega$, customarily defined so that at periastron the secondary star is moving away from us at $\omega=180^{\circ}$ and is on the far side of 
the primary at $\omega=270^{\circ}$. As noted above, the intrinsic X-ray temperature can be used to constrain the speed of one (or both) of the stellar winds. The intrinsic X-ray temperature should vary little, because it depends chiefly on $T$ and therefore on the wind speed $v_{2}$, which we expect to be more or less constant.

\section{Modeling the RXTE X-ray Lightcurve of $\eta$ Car}

The first attempts $[51,52,88]$ to explain the RXTE X-ray lightcurve of $\eta$ Car with colliding wind models were reasonably encouraging. Simple analytic models could describe the overall variation in X-ray flux and in $N_{H}$ and the observed constancy of temperature $T$ with plausible assumptions about the stellar winds, component masses and orbital inclination. The abrupt change from $X$-ray maximum to minimum is suggestive of an eclipse of the X-ray source region by the primary star's wind, so these first attempts assumed that the $X$-ray minimum was due entirely to an increase in absorbing column $N_{H}$, using simple, 1-dimensional, spherically symmetric wind models. Since the duty cycle of the X-ray "eclipse" is so small, it is very likely that the $X$-ray minimum occurs near periastron, when the stellar separations and orbital geometry are changing most rapidly and column densities should naturally be large as the $X$-ray source becomes surrounded by the thick inner primary wind. These initial attempts required a high eccentricity $(e \approx 0.9$, similar to the eccentricity of WR 140) and were consistent with an orientation in which the companion star is in front of the primary near apastron and behind at periastron. However, because the orbital geometry changes so rapidly around periastron, the duration of the X-ray minimum in these simple "eclipse" models was always far shorter than the observed duration. There are many modifications possible to correct this, among them:

- The parameters $i$ and $\omega$ can be altered $[26,30,53,70]$;

- Tidal and other effects may suddenly increase the primary wind's density near periastron $[23,26,81,11,61]$;

- The large-scale structure of the primary wind is not spherical [81];

- The orientation of the shock structure may be significantly distorted by orbital motion [74, 70];

- Radiative cooling may destabilize the shock near periastron, possibly causing a general disruption or collapse of the $X$-ray source region $[30,61]$;

- and other effects $[87,37,82,83]$, including radiative inhibition of the winds near periastron 721

Most of these possibilities are not mutually exclusive; some of them appear quite likely, several may occur together, and some can reinforce each other.

Throughout the cycle the observed $X$-ray flux depends critically on absorption in the intervening part of the dense primary wind; $N_{H}$ obviously varies with phase in the orbit. The wind geometry is complex, since the secondary wind produces a cavity on one side of the primary wind and orbital motion affects the cavity's orien- 

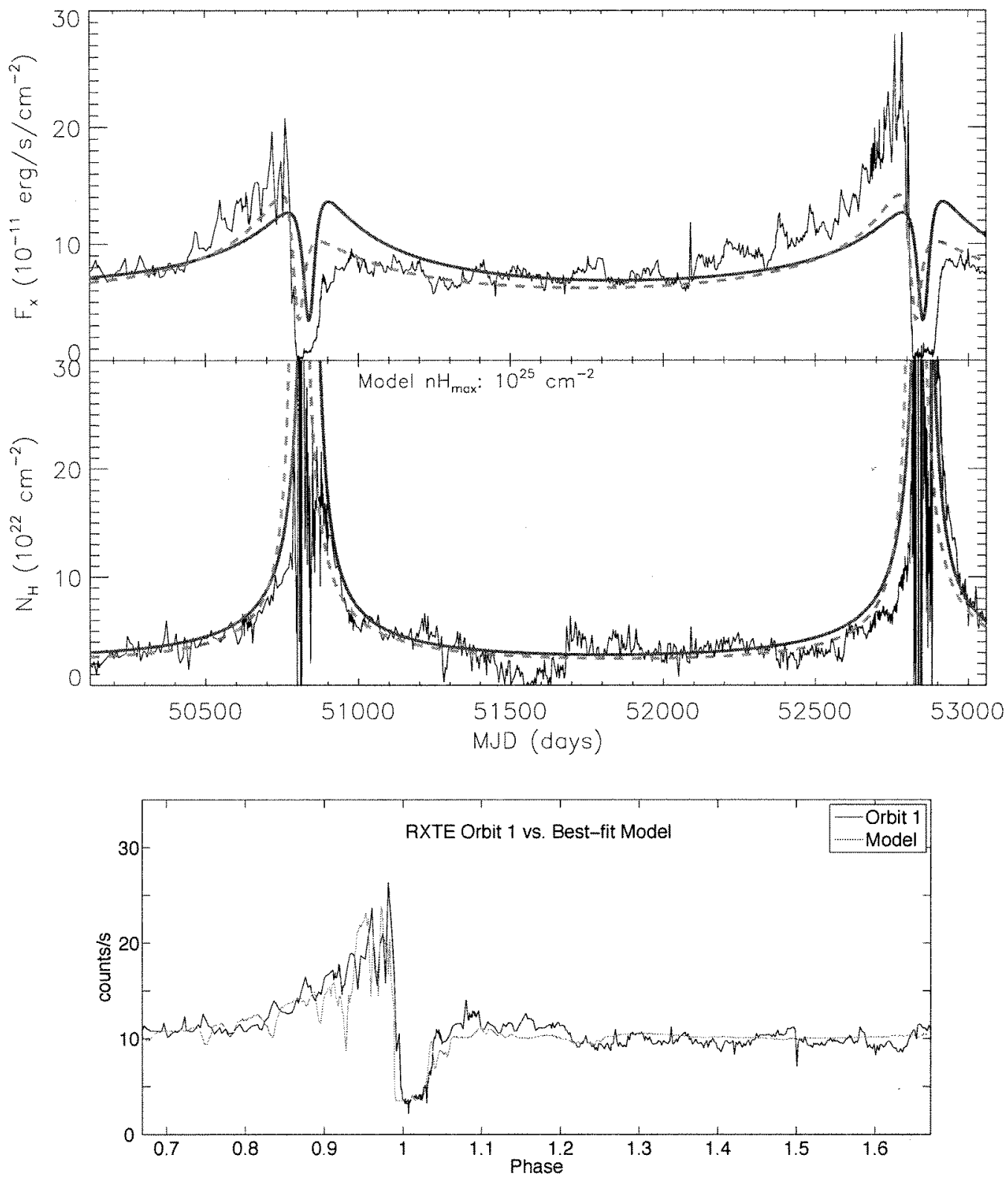

Fig. 4 Modeling the RXTE X-ray lightcurve of $\eta$ Car. Top: The solid blue line shows an estimate[52] of the X-ray light curve using an analytical model[92] with published orbital parameters [17], assuming that the $\mathrm{X}$-rays originate from a point near the apex of the shock cone. The dotted red line shows the results of the same analytic model based on more recently published orbital elements 707 . Millle: Variations in column depth $N_{H}$ to the X-ray source along with the model variation[52]. Line colors are as above. Bontom: X-ray lightcurve (black) and model (red) based on a 3-D smoothed particle hydrodynamics model[70] which better accounts for the variation in column depth from the wind of the primary and successfully recovers the depth and duration of the X-ray minimum. None of the models account for observed cycle-to-cycle changes. The two upper panels are adapted from Ishibashi et al. [52], and the lower panel is from Okazaki et al. 170]. 
tation and shape. Analysis of the X-ray spectrum near the mid-point of the cycle[74] (i.e. near apastron in the orbit) showed that the velocity of the companion's wind is $\approx 3000 \mathrm{~km} \mathrm{~s}^{-1}$, while its wind mass-loss rate is $\approx 10^{-5} \mathrm{M}_{\odot} \mathrm{yr}^{-1}$. Recent efforts to model $\eta$ Car's colliding winds with gas-dynamic simulations $[74,70,72]$ attempt to account for variations in the large-scale wind geometry. Dynamical wind models [71] and smoothed-particle simulations [70] show the distortion of the wind by the secondary star's orbital motion. Fig. 5, for instance, shows a smoothedparticle model of the wind-wind interaction at a number of orbital phases [70]. A post-periastron buildup of material on the forward-facing shock surface causes extra absorption that extends the eclipse. Thus a pure eclipse model with suitable parameters can account for the duration of the X-ray minimum [74, 70], but apparently only if the X-ray emitting region is small. If the X-ray emitting region is extended, the eclipse model has difficulty reproducing the extended duration of the $\mathrm{X}$-ray minimum[72]. This does not disprove the complications and alternative models listed above, however; observations in other wavelength regions, beyond the scope of this review, are harder to explain than the X-ray minimum [61]. In summary, at phases of the cycle not near periastron, i.e., not during a spectroscopic event, the models cited above are probably realistic in a semi-quantitative sense. They may be valid near periastron as well, but the complexity of the situation there makes this difficult to establish.

Altogether, then, one can attempt to fit most of the X-ray "light curve" with just two orbital parameters, $e$ and $\omega$, and the mass-loss rate and terminal velocity of the companion star's wind. In 2000 Ishibashi estimated $e \approx 0.8$ and $\omega \approx 200^{\circ}$ by this method [53]. Implicitly using the same dependences, more elaborate calculations by Okazaki have led to $e \approx 0.9$ and $\omega \approx 243^{\circ}[70]$. Thus, if the reasoning is valid, periastron occurs $20^{\circ}$ to $70^{\circ}$ before "superior conjunction" (the point where the secondary star is most nearly on the far side of the primary). Orientations in which the companion star is in front of $\eta$ Car at periastron have also been suggested [34], but these require either a very large buildup of absorbing material behind the X-ray source or a dramatic change in the shock emissivity near periastron passage.

\section{Dynamics of the X-ray Emitting Region}

Thermal colliding-wind emission is rich in $\mathrm{X}$-ray lines that provide information on flow dynamics (via line widths and velocities), abundances (from line strengths) and temperatures and densities (from line ratios). The strongest lines are $\mathrm{K}$-shell transitions in hydrogen-like and helium-like ions. In the case of $\eta$ Car's wind, the observable X-ray features are broad lines of Si XIII, Si XIV, S XV, and S XVI, and Fe XXV at higher energies [41]. The implied elemental abundance ratios appear to be near solar $[8,41]$, which is not surprising since neither $\eta$ Car nor its companion star is thought to be evolved enough to show enhanced silicon, sulfur or iron abundances at the surface. Unfortunately the carbon, nitrogen, and oxygen abundances, 
which help trace the evolutionary state of the system, are all at energies below $1 \mathrm{keV}$ and so cannot be studied this way because of circumstellar absorption.

Observations in 2000-2003 with the transmission gratings on the CHANDRA Xray telescope have resolved the broad lines and show that they apparently vary with phase in the 5.5-year cycle [41]. Near times of X-ray minimum these lines shift to increasingly negative Doppler velocities while their profiles become broader and more distorted $[3,41]$. Interpretations of these changes are controversial. The observed variations may represent changes in the spatial distribution of the hot shocked gas [41], or perhaps the appearance of an additional outflow in the system [3]. So far only a single cycle has been observed so it's not clear if these changes are secular or phase-dependent.

The spectrum also shows a $6.4 \mathrm{keV} \mathrm{K}$-shell line of less ionized species of iron $[8,12,40]$. This is presumably fluorescence produced as the main X-ray emission passes through "cold" iron rich material, either in the wind of $\eta$ Car or in the Homunculus. The fluorescence line is broad, suggesting that at least part of it originates in fluoresced material in $\eta$ Car's wind. During the minimum the line increases in equivalent width, but the measured increase is rather modest, which may mean either that the scattering zone is compact enough to be heavily obscured at that time, or else that the intrinsic X-ray emission actually declines during minimum.

Thomson-reflected X-rays from the Homunculus have been resolved in CHAN$D R A$ images during the minimum when the direct $\mathrm{X}$-rays are weak [13]. Such observations are important since $X$-ray reflection could (in principle) provide us with a 3-D view of the cold absorbing wind material as a function of orbital phase. Light travel time delays are a complication, since light travel time for the reflected $\mathrm{X}$-rays may be as much as 80 days towards the back-projected northwest lobe.

\section{The X-ray Spectrum During the Minimum}

The best measures of the $X$-ray spectrum during the 2003 minimum used the $X M M$ Newton and CHANDRA X-ray observatories [13, 40,41]. XMM's imaging detectors provide sufficient spatial resolution to exclude sources unrelated to $\eta \mathrm{Car}$, while spectral imaging with CHANDRA resolved the source even from contamination by the Homunculus and outer ejecta. These observations $[40]$ confirmed that the apparent X-ray flux decreased even at $10 \mathrm{keV}$, requiring either a column density $N_{H} \gtrsim 10^{25} \mathrm{~cm}^{-2}$ lasting for two months or more or else a real decrease in the intrinsic hard X-ray emission. Meanwhile, analyses of the XMM spectra [40] suggested that the relevant average column density increased by only a factor of 5 to 10 during the minimum, reaching a maximum of roughly $4 \times 10^{23} \mathrm{~cm}^{-2}$. This was not sufficient to explain the observed hard $\mathrm{X}$-ray decrease. Either the analysis underestimated the maximum $N_{H}$, or else the intrinsic X-ray production considerably decreased, i.e. the event was not merely an eclipse $[100,25,26]$.

During the minimum the hard X-ray flux varied strongly in timescales of days but not hours [40]; this seems reasonable if the lateral scale size of the emitting 
region was of the order of $1 \mathrm{AU}$ at that time. The minimum apparent flux in the 2$10 \mathrm{keV}$ band was only $0.7 \%$ of the maximum seen by RXTE. The slope of the X-ray continuum above $5 \mathrm{keV}$ does not vary much, indicating that the electron temperature of the hottest plasma does not vary significantly at any phase. The X-ray emission seemed to show two states [40] during the minimum: first a "deep" state at the start of the minimum, in which the entire $2-10 \mathrm{keV}$ flux was reduced, and then a "shallow" state beginning about midway through the minimum, when the $6-10$ $\mathrm{keV}$ emission increased and then remained constant for a brief period while the $E<6 \mathrm{keV}$ emission continued to increase as the column density decreases.

The 2003 minimum also revealed a previously hidden "central constant emission" component of X-rays [40]. This dominated the system brightness at energies $E<2 \mathrm{keV}$ during the minimum, with an intrinsic X-ray luminosity of $\sim 10^{34} \mathrm{ergs} \mathrm{s}^{-1}$ in the $0.1-10 \mathrm{keV}$ band. Its origin is unknown; if thermal, the observed temperature implies a flow velocity of $1000-2000 \mathrm{~km} \mathrm{~s}^{-1}$. It may represent the interaction of $\eta$ Car's polar wind with nearby structures, perhaps the "Little Homunculus" [54] (though it's not clear that the Little Homunculus would survive any such collision for long); or it might be emission produced by outflowing shocked plasma interacting with cooler circumstellar material. It might also be intrinsic emission produced by shocks distributed in one of the stellar winds.

\section{The Companion Star}

To date, there has been no generally accepted direct detection of the secondary $\operatorname{star}^{1}$, but for reasons noted below this is not surprising. In the absence of direct UV-toIR information, the $\mathrm{X}$-ray data provide perhaps the most useful (though indirect) constraints on its physical parameters.

First, even if we were unaware of the minima and the 5.5-year cycle, the X-rays strongly suggest that $\eta$ Car has a companion star. The average X-ray to total luminosity ratio, $L_{\mathrm{X}} / L_{\mathrm{bol}} \sim 5 \times 10^{-6}$, is very high for an evolved massive star, probably indicating that some sort of companion object is needed to help generate the X-rays. There's significant flux at high photon energies $E>5 \mathrm{keV}$, and such hard X-ray emission in evolved massive stars is almost unknown except in magnetized objects (whose $\mathrm{X}$-rays vary on short rotational time scales) and binaries.

In a colliding-wind model, the $\mathrm{X}$-ray temperature indicates the pre-shock speed of the wind; $\eta$ Car's X-ray temperature requires a wind speed of about $3000 \mathrm{~km} \mathrm{~s}^{-1}$ $[26,74]$. This is far higher than speeds observed in the primary wind. Indeed it is near the upper limit of wind speeds observed for any massive stars. For a radiatively driven wind from a hot $\left(T_{\text {phot }}>21000 \mathrm{~K}\right)$ massive star the wind terminal velocity is

\footnotetext{
Iping et al asserted that certain variable emission features in the $905-1180 \AA$ wavelength range were directly associated with the secondary wind [48]. This may be true but it is controversial $[44$, 61,811 . The reported features were much narrower than one would expect for the fast secondary wind, and in some models they can originate in the primary wind. The wind velocities derived by Iping et al. were also about a factor of $2-3$ too low to produce the colliding wind $X$-ray emission.
} 
related to the photospheric escape speed $V_{e s c}$ as $V_{\infty} \approx 3 \times V_{\text {esc }}$ [57], so that a terminal speed of $3000 \mathrm{~km} \mathrm{~s}^{-1}$ corresponds to a photospheric escape velocity of roughly $1000 \mathrm{~km} \mathrm{~s}^{-1}$. For a massive, luminous star the escape velocity is modified from the usual relation to account for acceleration due to radiation pressure on electrons: $V_{e s c}=\sqrt{2 G M(1-\Gamma) / R}$, where $M$ and $R$ are the mass and radius of the star, and $\Gamma$ the ratio of radiative Thomson acceleration to gravitational acceleration[57].

In order to produce the observed X-ray luminosity, the same models show that the wind mass loss rate of the secondary star must be $M_{2} \sim 10^{-5} M_{\odot} \mathrm{yr}^{-1}$. This is exceptionally high for an O-type star, but seems marginally allowable for an object above $40 M_{\odot}$. The determination of $\dot{M}_{2}$ is rather complex, depending on the poorly-constrained cooling ratio $t_{\exp } / t_{\text {rad }}$ discussed in Section 3 above. For example, inhomogeneities in the wind might increase $t_{\exp } / t_{\text {rad }}$. It's possible that improved models can attain the observed X-ray luminosity with a smaller value of $\dot{M}_{2}$.

In a "least surprising" evolutionary picture, one expects the companion object to be an early- or mid-O type star. Being less massive than $\eta$ Car it should be less evolved and therefore near the main sequence. A mid-O spectral type with $T_{\mathrm{eff}} \sim 35000 \mathrm{~K}$ would account for the photoionization seen in the ejecta $[25,26,28,43,48,47]$. Thus the most straightforward and most probable type of companion is a fairly "normal" star in the $30-60 M_{\odot}$ mass range, though other possibilities can be devised. Other indirect arguments based on photoionization of circumstellar clouds by the UV flux from the companion star al so point to a similar, though perhaps somewhat more evolved, object $[93,62]$. The recent analysis by Mehner et al. [62] demonstrated that an $04-06$ giant with $\mathrm{L} \sim 4 \times 10^{5} L_{\odot}, T_{\text {eff }} \approx$ $39,000 \mathrm{~K}$, and $\mathrm{M}_{\text {ZAMS }} 40-50 M_{\odot}$ would fit the observed range of parameters quite well. Such a star radiates mainly in the hard-to-observe far UV, should be less than $3 \%$ as bright as the primary at near-UV to IR wavelengths, and produces no spectral features that would be readily detected when superimposed on the complex primary wind spectrum [44]. The lack of a direct detection of the companion is therefore unsurprising. The only disquieting detail is the companion's high mass-loss rate required in some X-ray models, which might be viewed as evidence that the companion is a Wolf-Rayet star instead of an unevolved O star - making the system's evolutionary status problematic. However, we noted above that the deduced value of $\dot{M}_{2}$ is fairly uncertain.

\section{Other Unresolved Issues}

\section{$8.1 X$-ray Flaring}

The RXTE/PCA count rates (Fig. 2) show occasional brief maxima, which become dramatic in the year before the X-ray minimum. Early in 1997 Ishibashi noticed that the brightest X-ray "flares" tended to recur every 85 days [50]. This periodicity was 


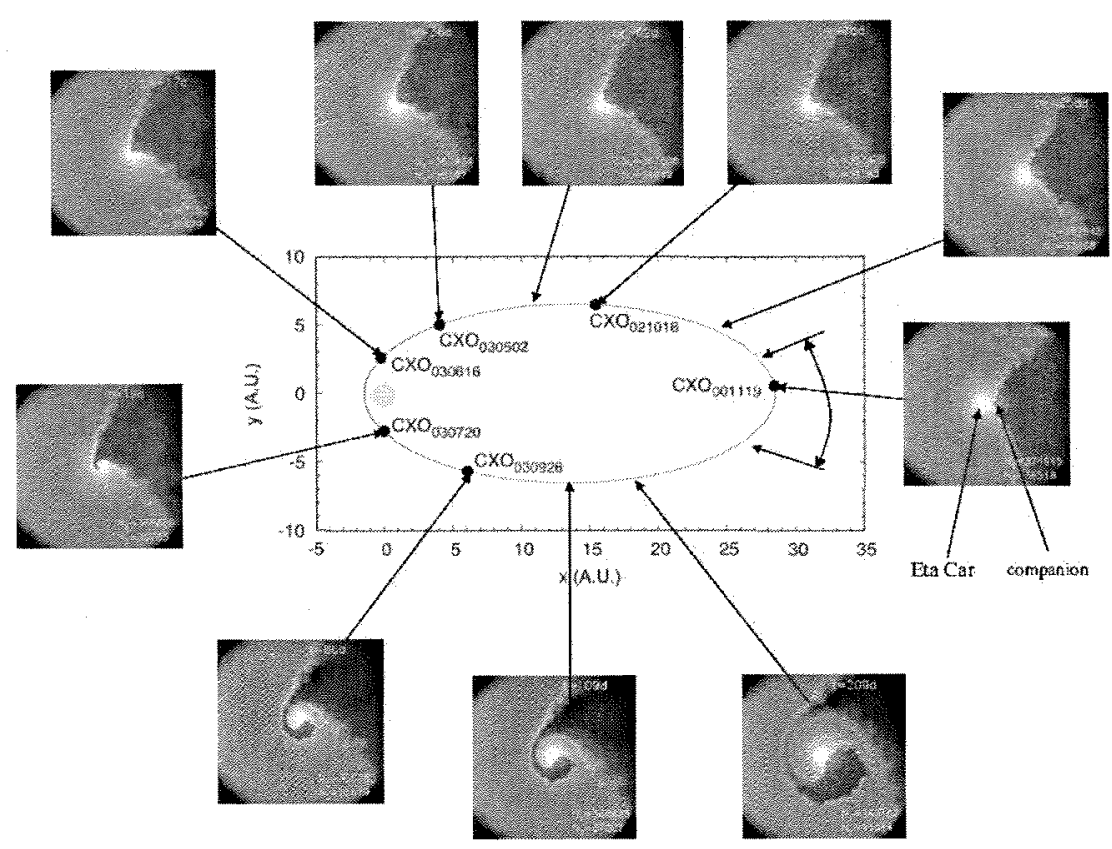

Fig. 5 Possible orbit of companion star. The orbit has an eccentricity $e=0.9$ with semi-major axis $a \approx 15$ AU. Dots along the orbit show CHANDRA (CXO) observations, while images show the projected density in the orbital plane of the interacting winds of $\eta \mathrm{Car}$ (high density; yellow-red) and the companion (lower density, blue-black) in one type of 3-d model [70]. The curved arrowed line shows the best guess of the orientation of the line of sight projected onto the orbital plane.

statistically doubtful, but it correctly predicted the next two major flares in July and September 1997, and perhaps another in March 1998 after the X-ray minimum [51].

$\mathrm{X}$-ray spectra show that they represent increased values of the volume emission measure $[9,49,8]$, though some hardening of the spectrum during flares has been noted $[14,40]$. The evidence so far favors a change in the hot colliding-wind plasma as the cause, rather than sporadic decreases in intervening absorption $[9,49]$. The physical mechanism producing the $\mathrm{X}$-ray flaring is not understood. In a simple qualitative model, localized density enhancements (clumps, or structured entities such as co-rotating streams [66]) in the wind eventually encounter the wind-wind shock and provide a brief increase in the amount of shocked X-ray emitting material. The flow timescale through the shock is $t_{\text {flow }} \sim a / v_{\text {flow }}$ where $a$ is a characteristic size (thickness?) of the shocked region and $v_{\text {flow }}$ is presumably a few hundred $\mathrm{km} \mathrm{s}^{-1}$ based on the wind speeds, or roughly 0.1 to $0.4 \mathrm{AU}$ per day. The observed 10-30 day flare durations are consistent with emission-region sizes of several AU [65].

The nature of the 85-day "quasi-period" is still not understood. A recurrence timescale of 85 days seems reasonable for either rotation or pulsation of the primary 
star; and in some models it might vary from cycle to cycle if each spectroscopic event alters the star's outermost structure [31]. But the interval between flare peaks varies with $\mathrm{X}$-ray cycle phase, decreasing to as little as 20 days just before $\mathrm{X}$-ray minimum, qualitatively consistent with expectations based on flow travel times [24].
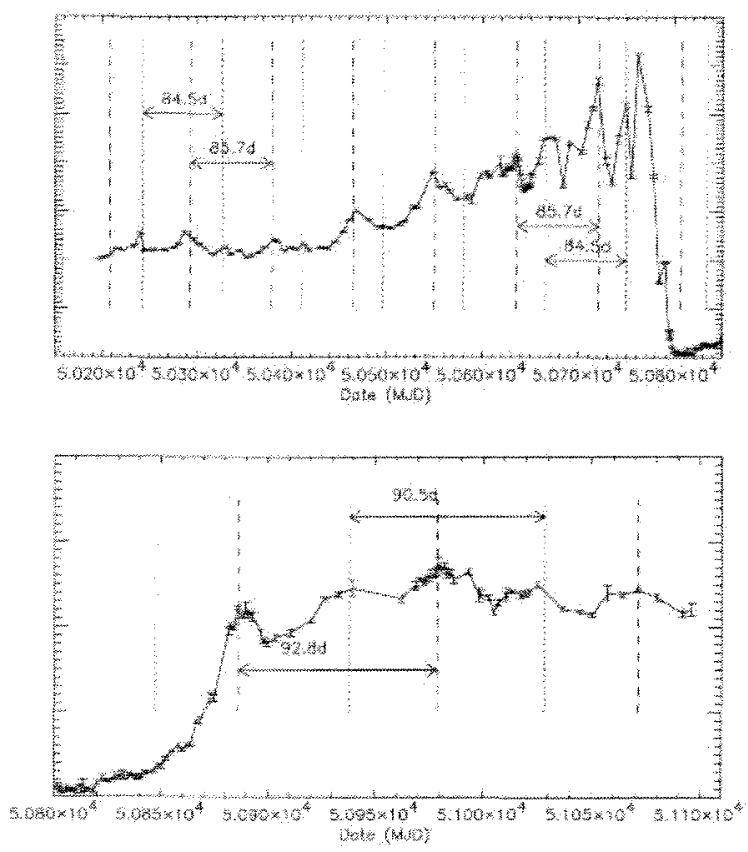

Fig. 6 Timing of major and minor X-ray flares in 1997-1998 according to Ishibashi [49].

\subsection{Cycle-to-Cycle Variations}

$\mathrm{X}$-ray monitoring also revealed significant variations in the average $\mathrm{X}$-ray flux level from cycle to cycle. Figures 2 and 7 shows RXTE count rates in the three 5.5-year cycles.The first and third cycles had very similar flux levels, but cycle 2 was significantly brighter. In a standard colliding-wind model the flux depends solely on orbital parameters, stellar mass-loss rates, and wind speeds (Section 3). Among these the mass-loss rate is probably most likely to change without perceptibly affecting the shape of the $\mathrm{X}$-ray spectrum.

Cycle-to-cycle changes have also been observed in other wavelength bands. He I $10830 \AA$ emission showed a secular decrease for several cycles [16], centimeterwavelength radio fluxes have been brighter in 2004-2008 than they were in 19982003 (S. White, priv. comm.), and the profiles of the extremely bright $\mathrm{H} \alpha$ and $\mathrm{H} \beta$ 
emission lines conspicuously differed between the 1997 and 2003 events [32]. Altogether, these developments likely indicate some undiagnosed change in the primary wind, although variations in other wavebands may be more complicated to interpret because they can be heavily influenced by secular changes in the Homunculus produced by expansion of the nebula and/or the creation or destruction of dust.

The most recent X-ray minimum which began on 16 January 2009, Figure 7, was consistent with the phasings of the two previous minima. However, the X-ray recovery began on 18 February 2009, one month earlier than expected based on the duration of the two previous X-ray minima. During the 2009 recovery the X-ray hardness increased markedly, similar to the spectral change which occurred at the end of the previous two minima, although the maximum hardness observed in 2009 was less than the maximum observed after the previous recoveries. This implies some fundamental change in the properties of the colliding wind emission. This may involve a decline in $\eta$ Car's mass loss rate $[55,63,15,64]^{2}$, though changes in the wind properties of the companion cannot be completely ruled out at this point.

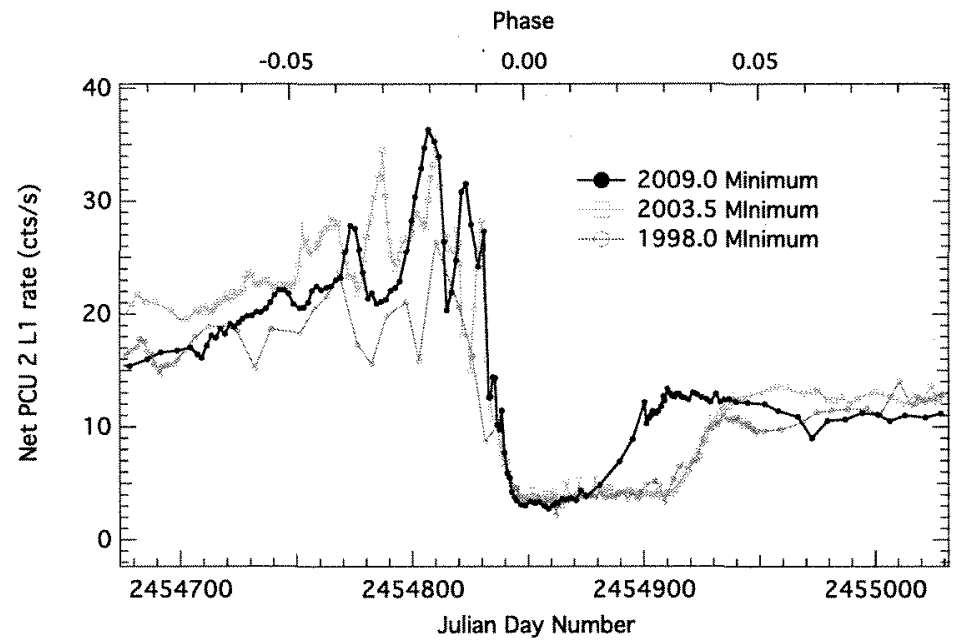

Fig. 7 Comparison of three X-ray minima observed by RXTE. The start of the 2009 minimum (16 January 2009) was consistent with the ephemeris derived from the previous two minima. The bottom axis shows the Julian Day Number corresponding to the 2009 minimum. The $X$-ray flux recovery occurred about 30 days early compared to recoveries from the previous two minima. From Corconan et al. [15].

${ }^{2}$ See Figure 10 in the Humphreys and Martin chapter. 


\subsection{The Cause of the X-ray Minimum}

The X-ray minimum may be caused by an increase in the intervening absorption, a decrease of the intrinsic emission, or both; in either case the maximum temperature is observed to be fairly constant $[52,40]$. All models so far agree that the event happens near periastron and, if an eclipse plays a leading role, the eclipsing agent is the primary wind and not the primary star itself.

But several pieces of the puzzle do not easily fit into a simple eclipse model. The duration of the $\mathrm{X}$-ray minimum is much longer than predicted in simple eclipse models, though more realistic wind models do better jobs in matching the eclipse duration $[74,70]$. If the $\mathrm{X}$-ray minimum really occurs near periastron, simple models suggest that the intrinsic $X$-ray luminosity should be in excess of $10^{35}$ ergs s $^{-1}$ at $E>5 \mathrm{keV}$ at that time since $L_{x} \sim 1 / d$. As noted above, hiding high-energy emission this bright requires an enormous wind column density which is about a factor of 100 higher than the observed column density. Another problem is that, during the minimum, a fully hidden X-ray source should produce a fluorescence iron line with an equivalent width about a factor of 6 larger than the observed value [40]. Also, the $\mathrm{X}$-ray minimum shows two distinct phases (a deep stage and a shallow stage)[40], which is not expected in simple eclipse models.

Several non-eclipse ideas have been proposed. A spectroscopic event may be the sporadic ejection of extra material by the primary star, perhaps triggered by the companion star's close approach $[100,26,11,61]$. This causes various effects, including a sudden rise of X-ray absorption. The latitudinal structure of the primary wind may suddenly change, increasing the wind density at equatorial latitudes where the secondary star is probably located [81]. Near periastron, the wind-wind shocks may disintegrate, collapse, or at least become very complex because of instabilities that arise when radiative cooling dominates $[30,61]$. This would destroy most of the highest-temperature regions, thereby replacing the $2-10 \mathrm{keV}$ emission by unobservable soft $X$-rays. Breaking the shock apart by accretion of part of the primary wind onto the companion star [83] could also reduce the colliding wind emission near periastron. Near periastron, the secondary star might move in to a dense structure such as a disk around the primary. These possibilities are not all mutually exclusive; in fact the first, second, or fifth of them would make the third more likely. Nor are they inconsistent with an eclipse. which, based on the likely orbit orientation, is likely to begin shortly thereafter [61].

The near-constant slope of the continuum above $5 \mathrm{keV}$ is evidence that the highest temperatures are not reduced during periastron passage [40]. Finally, the strange He II $\lambda 4686$ emission, which peaked in a dramatic way during the 2003.5 event, seems directly pertinent to the $\mathrm{X}$-rays for two reasons: it rose quickly in brightness during exactly the time when the $2-10 \mathrm{keV}$ brightness was fading, and most likely it requires a remarkably increased fux of soft $X$-rays below $0.5 \mathrm{keV}$, which of course cannot be observed directly [61]. 


\section{Future Prospects}

$X$-ray observations provide unique direct information on the mass flows in the 7 Car system and indirect information about the system and stellar parameters. The recent $X$-ray minimum in January 2009 has been observed by a lleet of $X$-ray observatories including RXTE, CHANDRA, XMM, Swift and Suzuku with a primary goal of constraining the flow dynamics and determining the nature of the $X$-ray minimum. In the coming years high resolution $X$-ray spectra with $X$-ray calorimeters on observatories like the Astro-H satellite [90] should resolve the He-lke iron $\mathrm{K}$-line triplet at $6.7 \mathrm{keV}$ for the first time and provide well-defined profiles of the the H-like iron line near $7 \mathrm{keV}$. These observations will be important since the high formation temperature of these lines mean that they must be formed near the stagnation point of the wind-wind collision; thus the line centroids can in principle be used to trace the orbit of the stagnation point, which can in turn be directly related to the radial velocity curve of the companion. This would enable us to dynamically constrain the system mass function and provide key answers about the evolution of the binary. Resolving the He-like iron $\mathrm{K}$ line triplet also provides important density and temperature diagnostics of the shocked gas near the stagnation point.

Even higher-energy images of hard $X$-ray and Gammaray emission from $\eta$ Car have recently been reported[59,89,2], using modern observatories like the Intemational Gamma-Ray Astrophysics Laboratory (INTECRAL), the Italian-led Gammaray satellite observatory Astrowivelatore Gamma a Immagini LEggero (Agile), and the Fermi Gamma-Ray Space Telescope. As history shows, high-energy high-spatial resolution observations are extremely important, and revolutionary technology like the proposed space-based $X$-ray interferometer[38] which could reach resolutions of micro-arcseconds at X-ray energies, holds out the hope of directly measuring the geometry of the colliding wind shock as it moves around the orbit.

These observations will provide much of the the crucial information (system masses, stellar parameters) needed to fully understand the evolution of $\eta$ Car. Looking even further down the road, $X$-ray observations will play an important role in understanding the eventual core-collapse of the star and the formation of a black hole and its associated supernova remnant.

Acknowledgements The anthors would like to acknowledge many frutful discussions with a large number of colleagues, anong them Kris Davidson. Ted Gull. Roberta Humphreys, Augusto Damineli, Jean Swank, Stephen White, Julian Pittard, David Henley, Tony Moffat, Stan Owocki, John Hiller, Virpi Niemela, Nolan Walbon, and many others too numerous to fully mention. This work was supported in part by Chandra grant GO3-4008A and NASA grant NAS5-32490. This research made use of software and data avalable from the High Energy Astrophysics Science Archve Research Center, and NASA's Astrophysies Data System.

\section{References}

1. Abbot, D. C. The terminal velocites of stollar winds fron early-type stars. Apl $225893+901$ 
(1978)

2. Abdo, A. A., et al.: Femi/Large Area Telescope Bright Gamma-Ray Source List. ApI Suppl $18346-66(2009)$

3. Behar, E., Nordon, R, and Soker, N.: A High-Velocity Transient Outflow in $\eta$ Carmae, Apl Letters 666 L97-L100 (2007)

4. Bradt, H. V., Rothschid, R. E., and Swank, J. H.: X-ray timing explorer mission. A\&A Suppl $97355-360(1993)$

5. Cherepashchuk, A. M. Detectability of Wolf-Rayet binaries from X rays. Soviet Astronomy Letters $2138+(1976)$

6. Chlebowski, T., Seward, F. D., Swank, J, and Szymkowiak, A.: Xurys from Eta Carina. ApJ $281665-672(1984)$

7. Corcotan, M.F., Rawley, G. L. Swank, I, H. and Petre, R.. First detection of X-nay variability of eta carinae. Apl Letters 445 L121-L124 (1995)

8. Corcomn, M. F., Petre, R., Swank, J. H., Drake, S. A. Koyana, K., Tsuboi, Y., Viotti, R., Damineli, A., Davidson, K., Ihibashi, K., White, S., and Curre, D.. The ASCA X.Ray Spectrum of eta Carinae. Apl $494381+(1998)$

9. Cowcona, M. F., Ishibashi, K., Davidson, K.. Swank, J. H. Petre, R., and Schmit, J. H. M. M. Increasing X-ray emissions and periodic outburst from the massive star Eta Carinae. Nature $390587+(1997)$

10. Corcoran, M. F., Fredericks, A. C., Petre, R., Swank, J. H., and Drake, S. A.: The X-Ray Spectral Evolution of $\eta$ Carinue as Seen by ASCA. Apl 545 420-428 (2000)

11. Corcotan, M. F, Ishibashi, K., Swank, J. H, and Petre, R: The X-Ray Light Curve of $n$ Carinar Refinement of the Obit and Evidence for Phase-dependent Mass Loss. ApJ 547 $1034-1030(2001)$

12. Corcomn, M.F., Swank, J. H., Petre, R., Ishibashi, K., Davidson, K., Townstey, L., Smith, R., White, S., Viott, R, and Damineli, A.. The Chandra HETCS X-Ray Grating Spectrum of $\eta$ Carinae. Apl $5621031-1037(2001)$

13. Corcotan, M.F, et a1.: Waiting in the Wings: Reflected X-Ray Emission from the HonuncuIus Nebula. Apt $613381-386(2004)$

14. Cowotan, M. F. X-Ray Monitoring of $\eta$ Carinat: Variations on a Theme. Al 129 2018-2025 (2005)

15. Corcoran, M. F, Hamaguchi, K., Pittare, J. M., Russell, C. M. P., Owocki, S. P., Parkin, E. R., Okazki, A.: Recent X-ray Variability of $\eta$ Carinae: The Quick Road to Recovery. Apl $7251528-1535(2010)$

16. Damineli, A.: The 5.52 Year Cycle of Eta Carinae. Ap Letters $460 \mathrm{L49}+(1996)$

17. Dammeli, A., Conti, P.S., and Lopes, D. F.: Eta Carinac: a long period binary?. New Astronony $2107-117(1997)$

18. Damineli, A., Lopes, D.F, and Conti, E.S.: Eta Carinae Binarity after the 1998 Event. ASP Conf. Ser. $179288-294$ (1999)

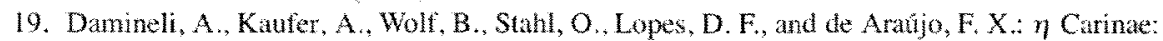
Binarity Contimed. Apl Letters 528 L101-L104 (2000)

20. Damineli. A., et al: The periodiciry of the n Carinat events. MNRAS 384 1649-1656 (2008)

21. Damineli, A., et al. A multispectral view of the periodic events in $\eta$ Carinae. MNRAS 386 $2330-2344(2008)$

22. Davidson, K. and Humphreys, R. M.: Eta Carinae and Its Enviroment. Ann. Rev. Astron. and Astrophys. 35 1.32 (1997)

23. Davidson, K.: Is Era Carinae a Long-period Binary? New Astronomy $2387+$ (1997)

24. Davidson. K., Ishibashi, K. and Corconn. M. F.: The relationship between two periodicities observed in Eta Carnae. New Astronomy $3241-245$ (1998)

25. Duvidson, K., Ishibash, K., Gull, TR., and Humphreys, R.M.: HSTSTIS Observations of the Star During Its Recent Event. ASP Conf. Ser. $179227-235$ (1099)

26. Davidson, K.: Why the Binary Hypothesis Isn't a Panacea. ASP Conf. Ser. 179304315 $(1999)$ 
27. Davidson, K., Ishibashi, K., Gull, T. R, Humphreys, R. M., and Smith, N. $\eta$ Carinae: Testing a Binary Orbit Model with the Hubble Space Telescope/Space Telescope Imaging Spectrograph. ApJ Letters 530 L107-L110 (2000)

28. Davidson, K.: Unique Spectroscopic Problems Related to Eta Carinue. ASP Conf, Ser. 242 $3-12(2001)$

29. Davidson, K. Smith, N. Gull, T. R., Ishibashi, K., and Hilier, D. I.. The Shape and Orientation of the Homunculus Nebula Based on Spectroscopic Velocities. AJ 121 1569-1577 (2001)

30. Davidson, K.: Chandra Meets Eta Carinat. ASP Conf. Ser. 262 267-274 (2002)

31. Davidson, K.: The Physical Nature of $\eta$ Carnae. ASP Conf. Ser. $332101+2005)$

32. Davidson, K., Martin, I., Humphreys, R. M., Ishibash, K., Gull, T. R., Stahl, O., Weis, K., Hillier, D. J., Damineli, A., Concoran, M., and Hamann, F.: A Change in the Physical State of $\eta$ Carinae? Ar $129900-906$ (2005)

33. Duncan, R. A.. White, S. M., Lim, J., Nelson, G. J, Drake, S. A., and Kundu, M. R.: Intense radio outbust from the supermassive star Era Carnae. Apl Letters 441 L73 L76 (1995)

34. Falcetaw Gonalves, D., Jatenco-Pereira, V., and Abraham, Z.: Wind-wind collision in the $\eta$ Carinae binary system: a shell-like event near periastron, MNRAS $357895-902$ (2005)

35. Feinstein, A. and Maraco. H. G.: On a possible three-year cycle of eta Car. A\&A 30 271-273 (1974)

36. Forman, W., Jones, C., Cominsky, L. Julien, P, Murray, S., Perers, G., Tananbaum, H., and Giacconi, R.: The fourth UHURU catalog of X-ray sources. ApJ Suppl $38357-412$ (1978)

37. Gayley, K. G., Owocki, S. P., and Crammer, S. R.: Sudden Radiative Braking in Colliding Hot-Star Winds. Apl $475786-(1997)$

38. Gendrean, K. C., Cash, W. C., Gorenstein, P., Windt, D. L., Karet, P., and Reynolds, C.: MAXIM: the black hole inager, $5488394402(2004)$

39. Criffths, R. E., Peacock, A., and Pagel, B. E. J.: Absence of soft X rays from eta Car. Nature $250714-715(1974)$

40. Hamaguchi, K., Corcoran, M. F., Gull, T., Ishbash, K., Pittard, J. M., Hiller, D. I, Damineli, A., Davidson, K., Nielsen, K. E., and Kober, G. V.: X-Ray Spectral Variation of 7 Carinae through the 2003 X-Ray Minimum. Apl 663 522-542 (2007)

41. Henley, D. B., Corcorn, M. F., Pittard, J. M., Stevens, I. R., Hamaguchi, K., and Gull, T. R. Chondra X-Ray Grating Spectrometry of $\eta$ Carine near X-Ray Minimum. I. Vuriability of the Sulfur and Silicon Emission Lines. ApJ $680705-727$ (2008)

42. Hill, R. W., Burginyon, G., Grader, R. J., Palmieri. T. M. Seward, F. D., and Stoering, J. P.: A Soft X-Ray Survey from the Galactic Center to VELA. ApJ $171519+(1972)$

43. Hillier, D. J., Davidson, K., Ishibashi, K., and Gull, T. On the Nature of the Central Source in $\eta$ Carinae. Apl $\mathbf{5 3 3} 837-860$ (2001)

44. Hiller, D. J., et al: The UV Scattering Halo and Central Source Associated with Eta Carinae. Apl $6421098-1116(2006)$

45. Hoffeit, D.: Elias and Francis Loomis on Variable Stars, 1866-69. Jourial of the American Association of Variable Star Observers (IAAVSO) $1367-77$ (1984)

46. Humphreys, R. M. and Davidson, K.: The Iuminous blue variables: Astrophysical geysers. PASP 1061025 1051 (1994)

47. Humphreys, R. M. Davidson, K., and Koppeman, M. The Early Spectra of Eta Carinae 1892 to 1941 and the Orset of Its High Excitation Emission Spectrum, AJ 135 1249-1263 (2008)

48. Iping. R. C., Sonneborn. G., Gull. T. R., Massa, D. L., and Hillier, D. J.: Detection of a Hot Binary Companion of $\eta$ Carmae. ApJ Letters $633 \mathrm{~L} 37 \mathrm{~L} 40$ (2005)

49. Ishbash. K. Multwavelength investigation of the supermassive star Eta Carinae (1999)

50. IShibashi, K., Davidson, K., Corcoran, M. F, Swank, I. H. Petre, R., and Jakoda, K.: Eta Carinae. LU Circ $66681+(1997)$

51. Ishibashi, K., Corcorm. M. F., Dwidson, K., Drake, S. A., Swank, J. H., and Petre, R.: Rew solving X-ray Temporal Variations in Eta Curmae. ASP Conf. Ser. 179. 266-274 (1999)

52. Ihhbashi, K., Corcoran, M. F, Davicon, K. Swank, J. H., Petre, R., Drke, S. A., Damineli, A., and White, S.: Recurrent X-Ray Emission Variations of $\eta$ Carinae and the Binary Iypothesis. Aul $524983-987(1999)$ 
53. Ishibashi, K.: Searching for Physically Acceptable Parameters for Ea Carinae's Conpanion. ASP Conf. Ser. $24253 \times 54$ (2001)

54. Ishibashi, K.. et al. : Discovery of a Litte Homunculus within the Homunculus Nebula of $\eta$ Carinae. A.I $1253222-3236(2003)$

55. Kashi, A and Soker, N.: Explaning the Early Exit of Eta Carinae from its 2009 X-Ray Mininum with the Accretion Model. Apl Letters 701 L59-L62 (2009)

56. Koyama, K., Astoka, I. Ushiman, N. Yamauchi. S., and Corbet, R. H. D.: Hard X-ray emiscion from the Carina Nebula. Apl 362 215-218 (1990)

57. Kudrizki, R.-P. and Puls, J.: Winds from Hot Sturs. Am. Rev. Astron and Astrophys 38613 $666(2000)$

58. Landau, L.D., and Lifshitz, E.M.: Fluid Mechanies, 2nd edition. Oxford: Pergamon (1987)

59. Leyder, J, C. Water, R., and Rauw, $G$.: Hard $X$-ray emission from $\eta$ Carinae, A\&A 477 L29-L32 (2008)

60. Lomis, E. On the Feriod of $\eta$ Argus. MNRAS $29298+(1869)$

61. Martin, J, C., Davidson, K., Humphreys, R. M., Hillex, D. J., and Ishibashi, K.: On the He II Emission in 7 Carinae and the Origin of lts Spectroscopic Events. ApJ 640474490 (2006)

62. Mehner. A. Davidson. K, Ferland, G. J., and Humphreys. R. M.: Highexcitation Emission Lines near Eta Carine, and Its Likely Companion Star. ApJ 710729742 (2010)

63. Mehner, A., Davidson, K. Humphreys, R. M. Martin, J, C., Feriand, G. J, and Walbom, N. R.: A Sea Change in Eta Cannae. Apl Leters 717 L22-L25 (2010)

64. Mehner, A., Davidson, K, Martin, J. C. Humphreys, R. M., Ishibashi, K., Ferland, G. I.: Critical Differences and Clues in Eta Car's 2009 Event. ApJ in press (2011)

65. Moffat, A.F.J. and Comoran, M. F. Understanding the X-tay Flaring from $\eta$ Carinae Apl $707,603-704(2009)$

66. Mullan, D. J.: Displaced narrow absorption components in the spectra of mass-losing OB stars - Indications of corotating interaction regions? A\& A $165157-162$ (1986)

67. Naze, Y, Corconan, M. F., Koenigsberger, G., and Mofut, A. F. J.: First Detection of Phasedependent Collding Wind X-Ray Emission outside the Milky Way. Apl Letters 658 L25-L28 (20m)

68. Nielsen, K. E., Corcoran, M. F. Gul, T. R., Hillier, D. J., Hamaguchi, K., Ivarsson, S., and Lindler, D. J.: Eta Carinat Across the 2003.5 Minimum: Spectroscopic Evidence for Massive Binary Interactions. ApJ 660, 669-686 (2007)

69. Nugent, J. J., Jensen. K. A., Nousek, J. A.. Garmire, G. P., Mason, K. O., Walter, F. M., Bowyer, C. S., Stern, R. A., and Riegler, G. R.: HEAO A-2 soft X-ray source catalog. ApJ Letters $\$ 11-28(1983)$

70. Okazaki, A. T., Owocki, S. P., Russell. C. M. P., and Corcoran. M. F. Modelling the RXTE light curve of $\eta$ Carinae from a $3 D$ SPH simulation of its binary wind collision. MNRAS 388 L39-L43(2008)

71. Parkin, R. and Pittard, J. M. A 3D dynamical Model of the colliding winds in binary systems. MNRAS 388, 1047-1061 (2008)

72. Parkin, E. R., Pittard, J. M., Corcorm, M.F., Hamaguchi, K, and Stevens, 1. R.: 3D modelling of the colliding winds in $\eta$ Carinat - evidence for radiative inhibition. MNRAS 3941758 $1774(2009)$

73. Payne Gaposchkin, C.: The Galactic Novae. (1957)

74. Pittard.J. M. and Corcoran, M.F.: In hot parsuit of the hidden companion of eta Carinae: An $X$ ray detemination of the wind paraneters. A\&A 383636-647 (2002)

75. Pollock. A. M. T., Corcoran, M. F, Stevens, I. R., and Wiliams, P. M.: Bulk Velocities, Chemical Composition, and lonization Structure of the X-Ray Shocks in WR 140 near Periastron as Revealed by the Chandra Gratings. ApJ 629482498 (2005)

76. Prilutski, O. F. and Usov, V. V.: X rays hom Woll Rayet binaries. Soviet Astronomy $202+$ (1976)

77. Sewad, F. D., Fage, C.G., Tumer, M.J.L., and Pounds, K. A. X-ray soutces in the southern Miky Way. MNRAS 177 13P-20P(1976) 
78. Seward, F. D., Forman, W. R., Giacconi, R., Griffiths, R. E., Hamden, F. R., Jones, C, and Pye, J. P.: X-rays from Eta Carinae and the surrounding nebula. ApJ Letters 234 L55 L58 (1979)

79. Seward, F. D, and Chlebowsk, T. X-ray emission from the Carina Nebula and the associated early stars. Ap $256530-542(1982)$

80. Shore, S.N.: An Introduction to Astrophysical Hydrodynamics, ch. 4. San Dievo: Academic Press (1992)

81. Smith, N., Davidson, K., Gull, T. R., Ishbashi, K., and Hilier, D. J.: Latitude-dependent Erfects in the Stellar Wind of $\eta$ Carine. ApJ 586432450 (2003)

82. Soker, N: The Binarity of Eta Catinae and Its Similarity to Related Astrophysical Objects. ApJ 619, 1064-1071 (2005)

83. Soker, N.: Accretion by the Secondary in $\eta$ Carnae During the Spectroscopic Event. I. Flow Patuneters. ApJ 635 540-546(2005)

84. Stall, O. Weis, K., Bomans, D. J, Davidson, K, Gull, T. R., and Humphreys, R. M. A spectroscopic evert of $\eta$ Car viewed from diferent directions: The data and first results. A\&A 435 303-312(2005)

85. Steiner, J. E. and Damineli, A. Deteetion of He II $\lambda 4686$ in $\eta$ Carmae. Apl Letters 612 L133L136(2004)

86. Stevens, I. R., Blondin, J. M., and Pollock, A. M. T.: Colliding winds from early-type stars in binary systems. Ap 386 265-287 (1992)

87. Stevens, I. R. and Pollock, A. M. T. Stagnation Point Flow in Colliding Wind Binary Systems. MNRAS 269 226-(1994)

88. Stevens, I. R. and Pitard, J. M.: Ea Carine: A Colliding Wind Binary? ASP Cont. Ser. 179. $205 \times 303(1999)$

89. Tavani, ef al. Detection of Gamma-Ray Emission from the Eta-Carinae Region. ApJ Letters 698 L 142 L L 146 (2009)

90. Takahashi, T., and the NeXT team: The NeXT Mission. ArXiv enprints $807(2008)$

91. Tsuboi, Y., Koyama, K., Sakuno, M., and Petre, R.: ASCA Okxervations of Eta Carmae. Pub Ast Soc Japan $4985-92(1997)$

92. Usov, V. V.: Stellar wind collision and $X$-ray generation in massive binaries. Apl $389635-648$ (1992)

93. Vener, E., Buhweiler, F. and Gull, T. The Binarity of $\eta$ Carinae Revealed from Photoion ization Modeling of the Spectral Varability of the Weigelt Blobs B and D. ApJ $624973-982$ (2005)

94. White, S. M., Dunan, R. A., Lim, J., Nelson, G. J., Drake, S. A., and Kundu, M. R.: The radio source around Eia Carinae. ApJ 429 380-384 (1994)

95. Whitelock, P. A., Feast, M. W., Carter, B. S., Roberts, G., and Gluss, I. S.: The infrared spectrum and variability of Eta Carinae. MNRAS $203385-392$ (1983)

96. Whitelock, P. A., Feast, M. W., Koen, C. Roberts, G., and Carter, B. S.: Variability of EtaCarinae. MNRAS $270364+(1994)$

97. Whitelock, P. A., Feast, M. W., Marang, F., and Breedt, E.: The 2003 Shell Event in Eta Carinae. MNRAS $352447-456(2004)$

98. Willis, A. J, Schild, H., and Stevens, I. R.: ROSAT bbservations of y Velonum (WC $8+09$ ). I. The discovery of colliding wind X-ray emission.. A\&A $298549+(1095)$

99. Wolf, R.: Schteiben des Hern Prof. Woll, Directors der Stemwate in Zinich an den Herausgeber. Astronomische Naclurichten $6059+(1863)$

100. Zanella, R., Wolf, B., and Stahl, O.: Spectroseopy of the shell episode of Eta CAR (19811983). A\&A $13779-84(1984)$ 